\title{
Optimal consumption of polluting and non-polluting goods: The role of habits
}

\author{
Alfred Greiner
}




\title{
Optimal consumption of polluting and non-polluting goods: The role of habits
}

\author{
Alfred Greiner*
}

\begin{abstract}
We present a basic exogenous growth model with two consumption goods that differ as regards their contributions to environmental pollution. Allowing for habit formation of the polluting good, we show under which conditions habit formation raises the consumption of the clean good relative to the dirty one in the competitive economy. Further, we demonstrate when habit formation generates a lower steady-state pollution stock compared to the situation without habits. Finally, we determine the Pigou tax rates and we illustrate that the social optimum may imply a higher steady-state pollution than the competitive economy if the habit formation is sufficiently strong.
\end{abstract}

JEL: Q50, H23, C61

Keywords: environmental pollution, clean and dirty consumption, habits, optimal control

*Department of Business Administration and Economics, Bielefeld University, P.O. Box 100131, 33501 Bielefeld, Germany, e-mail: agreiner@wiwi.uni-bielefeld.de 


\section{Introduction}

An important aspect in solving the problem of environmental pollution, such as global warming for example, plays technical progress. A great step towards a cleaner environemt will be achieved once it succeeds to replace polluting methods of production by nonpolluting ones. In analyzing that topic, economists often consider energy production and study the question of how a less polluting production process can be implemented without leading to output and welfare losses.

Thus, switching from dirty to clean energy is the subject of a great many studies. For example, Hoel and Kverndokk (1996) present a resource model where the use of the resource generates negative externalities. There exists a non-polluting perfect substitute for the polluting resource, with the non-polluting backstop being available at a constant unit cost. Hoel and Kverndokk show, among other things, that it is optimal to extract the polluting resource even when its price is equal to the price of the non-polluting resource. In a more recent contribution, van der Ploeg and Withagen (2014) adopt the model by Hoel and Kverndokk and change two assumptions: They do not allow for a decay of greenhouse gases and they assume that capital must be built up to produce the final output. However, capital is not a perfect substitute for the energy input but, rather, there is a backstop that can perfectly substitute the non-renewable energy source. Energy is produced using a polluting non-renewable resource and a non-polluting renewable energy source that is available at a constant unit cost, as in Hoel and Kverndokk. They show that it is optimal to use only the polluting resource initially and, later on, only renewables, when the initial stock of the polluting resource is small. The lower the cost of the renewable, the more of the polluting energy source is left in situ and the sooner the only renewable phase starts. The models by Hoel and Kverndokk (1996) and by van der Ploeg and Withagen (2014) have the same structure: There is a polluting resource that can be perfectly substituted by a non-polluting one at a given cost, with both variables being control variables. Hence, their models belong to the general class of models analyzed by Krautkraemer (1998). 
Greiner et al. (2014) present a more elaborate framework with regard to the backstop technology. There, it is assumed that a capital stock must be built up first in order to produce the renewable energy. The model considers the decentralized market economy and derives optimal taxes and subsidies such that the market economy replicates the social optimum. It turns out that it can be optimal to not completely exploit the non-renewable resource that is used to generate energy, but, rather to leave a certain part in situ. The outcome depends on the efficiency of the backstop technology, i.e. of the renewable energy source, and on the initial stock of the non-renewable resource.

All of those contributions have in common that they adopt a supply side view. Indeed, there exist only very few studies that analyze the role of preference with respect to the choice between a clean and a dirty consumption good. One exception is the contribution by Scalera (1996) who assumes that the stock of pollution depends on aggregate production and on the amount of the dirty consumption good, whereas clean consumption does not pollute the environment. The paper derives the Pigouvian taxes that make the decentralized competitive economy replicate the social optimum. Further, the paper shows that the trade-off between economic activity and the environment is mitigated by the presence of the non-polluting consumption good. Another paper with clean and dirty consumption is the one by Orecchia and Tessitore (2011). Those authors analyze an endogenous growth model where they allow for a clean and dirty consumption good and demonstrate that the substitution of the dirty consumption good by the clean consumption good is not sufficient to reduce the pollution of the environment. Further, they show that an environmental Kuznets curve may arise in their framework.

One aspect that is not considered in those papers dealing with clean and dirty consumption is the role of habits that can arise by consuming dirty consumption goods over a longer period of time. ${ }^{1}$ A contribution in the economics literature, where the effects of habits in a growth model with environmental pollution have been studied, is the paper

\footnotetext{
${ }^{1}$ For a survey of habits in economics, see for example Hodgson (2004).
} 
by Ikefuji (2008). He demonstrates that ongoing growth in his model with habits is only possible if there is sufficiently rapid technical change that stimulates the incentive to invest. But that contribution only considers a hoogeneous consmption good and does not differentiate between clean and dirty consumption.

In this paper, our goal is to study the optimal allocation between clean and dirty consumption allowing for habits that arise from the consumption of the dirty good. We assume that the dirty consumption good has been consumed over a certain time period in the past, when a clean consumption good becomes available. The consumer, then, solves a new intertemporal optimization problem where he can choose between the clean and the dirty good. We analyze both the competitive economy and the social optimum.

The rest of the paper is organized as follows. Section 2 presents the general model with a clean and a dirty consumption good, where the dirty consumption good may build up a stock of habits. Section 3 first analyzes the competitive economy in which the planer does not take into account the externalities in solving the optimization problem and 4 , then, studies the social optimum. Section 5, finally, concludes the paper.

\section{The model with clean and dirty consumption}

We consider an economy with a clean and a dirty consumption good, where the consumption of the dirty good may build up a stock of habits. Habits in our model are to be seen as routines that are formed as a by-product of human behaviour. In our model, they result from consuming the dirty consumption good in the past. Habits positively affect the utility of the individual since they represent routines that make the individual act without having to devote mental resources to achieve a certain goal. Thereby, habits simplify everday life because new situations are dealt with automatically that, otherwise, would require awareness and attention. The latter go along with efforts, in economic terms costs, that can thus be avoided. In this respect, psychologists speak of automative 
thinking that is based on schemes, i.e. cognitive structures, which are the result of unintended learning processes (see Aronson et al., 2004). Therefore, the habits are beneficial for individuals and raise their utility. In this context, we should also like to point to the so-called perseverance effect that makes individuals stick to certain routines even if they have proven to be wrong that reinforces the effects of habits.

The household sector is represented by a continuum of infinitely lived homogenous households with household production. Each individual household has measure zero and the household sector has mass one. The utility function $U$ of the representative household is assumed to be given $b y^{2}$

$$
U(\cdot)=\frac{\left(C_{d}(1+H)^{\chi}\right)^{1-\sigma}-1}{1-\sigma}+\frac{C_{c}^{1-\sigma}-1}{1-\sigma}-D(P)
$$

with $C_{d}\left(C_{c}\right)$ dirty (clean) consumption, $H$ the stock of habits and $1 / \sigma>0$ the intertemporal elasticity of substitution of consumption. The parameter $\chi=\{0,1\}$ determines whether habit formation occurs (for $\chi=1$ ) or whether habits do not arise (for $\chi=0$ ). It should be noted that the term $(1+H)$ guarantees that habits exert a positive effect on the utility going along with the consumption of the dirty good even for $H<1$ (in the case of $\chi=1)$. Further, the function specified in equation (1) implies that clean and dirty consumption are perfect substitutes. Environmental pollution is denoted by $P$ and $D(P)$ denotes the damages resulting from the stock of pollution that reduce utility of the household.

It must also be pointed out that in the case of habit formation $(\chi=1)$, preferences are no longer intertemporally independent. In our model, the constant intertemporal elasticity of substitution $1 / \sigma$ determines whether the utility function displays distant or adjacent complementarity. The condition $1 / \sigma<1$ is sufficient for distant complementarity and the condition $1 / \sigma>1$ is necessary for adjacent complementarity. Even if neither of those conditions is necessary and sufficient at the same time we speak of distant (adjacent) if

\footnotetext{
${ }^{2}$ We neglect the time argument $t$ as long as no ambiguity arises.
} 
$1 / \sigma<(>) 1$ holds. From an economic point of view, distant (adjacent) complementarity or, more concretely complementarity between distant (adjacent) dates, means that a small increase of consumption at $t_{3}$ shifts consumption from $t_{2}$ to $t_{1}$ (from $t_{1}$ to $t_{2}$ ), with $t_{1}<t_{2}<t_{3}{ }^{3}$ Loosely speaking, distant complementarity means that the household prefers to have consumption smoothed over time, while adjacent complementarity implies that the consumer prefers to have bundles of the consumption good at nearby dates.

Habits in our model may arise as a side effect of cumulated past consumption of the dirty consumption good. Following the seminal paper by Ryder and Heal (1973), habit formation is described by the following equation

$$
H(t)=\gamma \int_{-\infty}^{t} e^{\gamma(s-t)} C_{d}(s) d s, \text { for } \chi=1
$$

The parameter $\gamma>0$ gives the weight attributed to more recent levels of dirty consumption in the process of habit formation. The higher $\gamma$ is, the larger is the weight given to more recent levels of dirty consumption compared to consumption flows further back in time. Differentiating equation (2) with respect to time leads to

$$
\dot{H}=\gamma\left(C_{d}-H\right), H(0)=H_{0}
$$

As mentioned above, environmental pollution negatively affects the utility of the household, with $D(\cdot)$ giving the damages. The function $D(\cdot)$ is specified as

$$
D(P)=P^{2} / 2
$$

The environmental pollution is modelled as a stock and evolves according to

$$
\dot{P}=\omega_{1} C_{d}+\omega_{2} C_{c}-\xi P, P(0)=P_{0}
$$

The constant parameters $\omega_{1}>\omega_{2} \geq 0$ give the contribution of one unit of the dirty and of the clean consumption good to environmental pollution, respectively, and $\xi>0$ reflects the ability of the environment to recover.

\footnotetext{
${ }^{3}$ See Ryder and Heal (1973) or Greiner (1998), p. 72-74, for a more detailed discussion.
} 
Production in our economy takes place with physical capital, $K$, as the input factor. The evolution of the capital stock can be written as

$$
\dot{K}=A K^{\beta}-p_{d} C_{d}-p_{c} C_{c}-\delta K, K(0)=K_{0}
$$

The term $Y=A K^{\beta}$ gives output as a function of the capital stock. Output can be used for investment and for the consumption of the clean and of the dirty good, where $p_{d}$ and $p_{c}$ denote the constant price of the dirty consumption good and of the clean consumption good in terms of output, the price of which is set equal to one. The parameter $\delta$ is the depreciation rate, $A$ is a constant technology parameter and $\beta$ gives the elasticity of output with respect to capital.

\section{The competitive economy}

With competitive economy, we refer to the situation where the household maximizes the discounted stream of utility subject to the resoutce constraint (6), but neglects the two externalities (3) and (5). The justification for that is that there are a great many households so that the pollution of each individual household is small so that the representative household does not take it into account in formulating its optimization problem. In equilibrium, however, where all households behave identically, the aggregate stock of pollution is built up as a by-product of consuming the polluting consumption good and affects utility of each household. As concerns habit formation, this process happens unconsciously so that the household is not explicitly aware of it and, therefore, does not take account of it in setting up its optimization problem.

We assume that the representative household has consumed only polluting goods in the past up to $t=0$ that has built up a stock of habit. At $t=0$, a non-pollluting consumption good becomes available and the household has to choose between the polluting and the non-polluting good. Denoting the rate of time preference by $\rho$, the optimization problem 
of the representative household can, then, be written as

$$
\max _{C_{d}, C_{c}} \int_{0}^{\infty} e^{-\rho t}\left(\frac{\left(C_{d}(1+H)^{\chi}\right)^{1-\sigma}-1}{1-\sigma}+\frac{C_{c}^{1-\sigma}-1}{1-\sigma}-P^{2} / 2\right) d t
$$

subject to (6). To solve the optimization problem, we set up the current-value Hamiltonian as

$$
\mathcal{H}^{s}=U(\cdot)+\lambda_{1}\left(A K^{\beta}-p_{d} C_{d}-p_{c} C_{c}-\delta K\right)
$$

with the utility function $U(\cdot)$ given by (1) and $\lambda_{1}$ the shadow price or co-state variable of physical capital. The necessary optimality conditions for an optimum are given by

$$
\begin{aligned}
\frac{\partial \mathcal{H}}{\partial C_{d}} & =0 \leftrightarrow C_{d}=\left(\frac{(1+H)^{\chi}}{\lambda_{1} p_{d}}\right)^{1 / \sigma}(1+H)^{-\chi} \\
\frac{\partial \mathcal{H}}{\partial C_{c}} & =0 \leftrightarrow C_{c}=\left(\lambda_{1} p_{c}\right)^{-1 / \sigma} \\
\dot{\lambda}_{1} & =(\rho+\delta) \lambda_{1}-\lambda_{1} \beta A K^{\beta-1}
\end{aligned}
$$

In addition, we require that the usual transversality condition $\lim _{t \rightarrow \infty} e^{-\rho t} \lambda_{1} K=0$ must hold.

In equilibrium, environmental pollution (5) and habit formation (3) occur and influence the evolution of the economy. Thus, the economy is completely described by the following four-dimensional system of differential equations, in case of habit formation $(\chi=1)$, and by the three-dimensional system if habits do not occur $(\chi=0)$,

$$
\begin{aligned}
\dot{\lambda}_{1} & =(\rho+\delta) \lambda_{1}-\lambda_{1} \beta K^{\beta-1} \\
\dot{K} & =A K^{\beta}-p_{d}\left(\frac{(1+H)^{\chi(1-\sigma)}}{\lambda_{1} p_{d}}\right)^{1 / \sigma}-p_{c}\left(\lambda_{1} p_{c}\right)^{-1 / \sigma}-\delta K, K(0)=K_{0} \\
\dot{P} & =\omega_{1}\left(\frac{(1+H)^{\chi(1-\sigma)}}{\lambda_{1} p_{d}}\right)^{1 / \sigma}+\omega_{2}\left(\lambda_{1} p_{c}\right)^{-1 / \sigma}-\xi P, P(0)=P_{0} \\
\dot{H} & =\chi \gamma\left(\left(\frac{(1+H)^{\chi}}{\lambda_{1} p_{d}}\right)^{1 / \sigma}(1+H)^{-\chi}-H\right), H(0)=H_{0}
\end{aligned}
$$

Before we analyze the asymptotic behaviour of this system, we study the effect of habit formation on clean and dirty consumption by looking at the consumption of clean goods 
relative to dirty goods, defined as $C_{r e l}=C_{c} / C_{d}$. The share of the clean consumption good relative to the dirty one is given by

$$
C_{r e l}:=\left(\frac{p_{d}}{p_{c}}\right)^{1 / \sigma}(1+H)^{\chi(\sigma-1) / \sigma}
$$

From equation (16) we can derive our first result regarding the effect of habit formation in proposition 1.

Proposition 1 In the competitive economy, the presence of habits raises (reduces) the consumption of the clean good relative to the dirty good if the preferences of the consumer are characterized by distant (adjacent) complementarity.

Proof: Follows immediately from (16) with $1 / \sigma<(>) 1$ characterizing distant (adjacent) complementarity.

Proposition 1 shows that the ratio of the clean consumption good relative to the dirty one will be higher in the situation when habit formation occurs, compared to the situation without habit formation, when there is distant complementarity. The reason for that outcome is that in the case of distant complementarity the marginal product of the dirty consumption good is smaller when habits are present compared to the situation without habit formation. This holds because with distant complementarity the consumer tends to smooth consumption over time. A lower marginal product of consumption reduces the ratio of dirty to clean consumption or, equivalently, raises clean relative to dirty consumption. In case of adjacent complementarity, the reverse holds. Then, habit formation raises the marginal product of the dirty consumption good so that the ratio of clean consumption relative to the dirty one becomes smaller. Finally, we should also like to point out that in the situation without habit formation, the ratio of clean to dirty consumption is completely determined by the price ratio of these two goods.

Before we study the transitional dynamics, we analyze the effect of habit formation on the steady-state stock of pollution. Proposition 2 gives the result. 
Proposition 2 For $\omega_{1}>\omega_{2}\left(p_{d} / p_{c}\right)$, habits reduce (raise) the steady-state pollution if the preferences are characterized by distant (adjacent) complementarity. In the case of $\omega_{1}<\omega_{2}\left(p_{d} / p_{c}\right)$ habits raise (reduce) the steady-state pollution if the preferences are characterized by distant (adjacent) complementarity.

Proof: See appendix A.

In order to understand and interpret proposition 2, we note that habits raise the optimal value of the clean consumption good relative to the dirty one in the case of distant compementarity (see proposition 1). Since total aggregate output at the steady-state is given and independent of the presence of habits, more clean consumption implies less dirty consumption. This tends to reduce pollution. However, the overall effect depends on by how much clean and dirty consumption are increased and reduced, respectively, and on the contribution to pollution of one unit of the dirty consumption good and of one unit of the clean consumption good, i.e. on $\omega_{1}$ and on $\omega_{2}$. From the budget constraint of the household at the steady-state, we know that the relative price $p_{c} / p_{d}$ determines by how much the dirty good declines when the clean good rises by one unit. If that ratio is larger than one or, equivalently, if $p_{d} / p_{c}$ is smaller than one, a rise of the clean consumption good always reduces pollution because of $\omega_{1}>\omega_{2}$. If, however, $p_{c} / p_{d}$ is small so that an increase of the clean consumption good by one unit only leads to a small decline of dirty consumption, the latter may not be large enough so that pollution at the steadystate rises, due to the higher consumption of the clean good that also contributes to pollution, although to a smaller degree. This is particularly relevant when the difference between $\omega_{1}$ and $\omega_{2}$ is only small, i.e. when the clean consumption good is only slightly less polluting than the dirty good. Note that in the extreme case of $\omega_{2}=0$, i.e. when the clean consumption good is not polluting at all, the presence of habits will always reduce steady-state pollution in the case of distant complementarity. The same holds when the clean consumption good is more expensive that the dirty good, which is expected to be the more relevant case in real-world economies. 
In the case of adjacent complementarity, the argumentation is just reverse to the one in the situation with distant complementarity. With adjacent complementarity, the presence of habits reduces the ratio of clean consumption relative to dirty consumption at the steady-state. This effect will raise steady-state pollution, unless the increase of the dirty consumption is very small, as clean consumption declines, due to a very high price of the clean consumption good relative to the dirty good.

Before, we analyze the social optimum, we will study the question of uniqueness and stability of the steady-state. Proposition 3 gives the result for the competitive economy without habit formation.

Proposition 3 In the competitive economy without habit formation, there exists a unique saddle point stable steady-state.

Proof: See appendix B.

Proposition 3 shows that our model is characterized by the usual long-run behaviour of this type of growth models, even in the presence of an environmental externality. Hence, there exists a unique value of the initial shadow price, $\lambda_{1}(0)$, giving unique values of initial clean and dirty consumption such that the economy converges to the long-run steady-state.

When we allow for habit formation the economy is described by a four-dimensional differential equation system. In this case, the economy is also expected to be characterized by a unique steady-state for plausible values of the intertemporal elasricity of substitution. Proposition 4 shows the result.

Proposition 4 In the competitive economy with habit formation, a sufficient condition for the existence of a unique steady-state is $1 / \sigma \leq 2$ and distant complementarity of the preferences, i.e. $1 / \sigma<1$, is sufficient for saddle point stability of the steady-state.

Proof: See appendix C.

Proposition 4 demonstrates that the model is not necessarily characterized by a unique 
saddle point stable steady-state when habits are allowed for. However, it must be pointed out that the conditions in the proposition are sufficient but not necessary for uniqueness and saddle point stability so that we can expect a unique saddle point stable steady-state even if they are not fulfilled. As regards the intertemporal elasticity of substitution, most of the empirical studies that estimate it find small values. However, there are substantial cross-country differences of this parameter and rich countries and economies with a high stock market participation substitute a larger fraction of consumption intertemporally in response to changes in expected asset returns. Havranek et al. (2015) perform a metaanalysis and find a mean of $1 / 2$ for the inter-temporal elasticity, but they also point out that the estimates vary greatly between economies. In particular, there are also countries for which the point estimate clearly exceeds 1 so that high values for that parameter cannot be considered as purely academic.

In order to illustrate our results derived up to now, we resort to a numerical example, where we use the following parameter values. We set the capital share to 30 percent, $\beta=0.3$, and the technology parameter is set equal to one, $A=1$. The prices of both consumer goods are set equal to one, $p_{c}=p_{d}=1$, and one unit of the dirty consumption good raises the stock of pollution by $0.1, \omega_{1}=0.1$, and is ten times as high as the contribution of one unit of the clean good, $\omega_{2}=0.01$. The depreciation rate of physical capital is 7.5 percent, $\delta=0.075$, and pollution recovers at a rate of $\xi=0.1$. The parameter $\gamma$ is set to 10 percent, $\gamma=0.1$, implying that the contribution of one unit of the dirty consumption good to current habits is $e^{-0.1 \cdot 1}=0.905$ and the contribution of dirty consumption five years back is $e^{-0.1 \cdot 5}=0.606$. Finally, the discount rate is set to 3.5 percent, $\rho=0.035$, and $1 / \sigma$ takes the value $1 / 2$ or $4 / 3$. Table 1 shows the steady-state values of the model without and with habit formation. ${ }^{4}$

\footnotetext{
${ }^{4} \mathrm{The}^{\star}$ denotes steady-state values.
} 


\begin{tabular}{|c|c|c|c|c|c|}
\hline & \multicolumn{2}{|c|}{ No habits $(\chi=0)$} & \multicolumn{3}{|c|}{ With habits $(\chi=1)$} \\
\hline & $C_{c}^{\star} / C_{p}^{\star}$ & $P^{\star}$ & $C_{c}^{\star} / C_{p}^{\star}$ & $P^{\star}$ & $H^{\star}$ \\
\hline $1 / \sigma=1 / 2$ & 1 & 0.673 & 1.243 & 0.613 & 0.545 \\
\hline $1 / \sigma=4 / 3$ & 1 & 0.673 & 0.844 & 0.719 & 0.663 \\
\hline
\end{tabular}

Table 1: Steady-state values of the competitive economy $\left(K^{\star}=4.192\right)$.

Table 1 demonstrates that the model with habits leads to a higher steady-state ratio of clean relative to dirty consumption in the case of distant complementarity $(1 / \sigma<1)$ and to a lower value of that ratio for adjacent complementarity $(1 / \sigma>1)$. Further, with distant complementarity the stock of pollution at the steady-state is lower compared to the model without habit formation because with both prices set equal to one, i.e. $p_{c}=p_{d}=1$, the condition $\omega_{1}>\omega_{2}\left(p_{d} / p_{c}\right)$ in proposition 2 always holds. With adajcent complementarity of the preferences, the presence of habits raises the steady-state stock of pollution. Finally, we should like to point out that for the parameter values underlying the outcome in table 1 , both model versions are saddle point stable with two and three negative real eigenvalues, respectively.

In the next section we present the social optimum and compare it to the competitive economy.

\section{The social optimum}

For the social optimum, the maximization problem is written as

$$
\max _{C_{d}, C_{c}} \int_{0}^{\infty} e^{-\rho t}\left(\frac{\left(C_{d}(1+H)^{\chi}\right)^{1-\sigma}-1}{1-\sigma}+\frac{C_{c}^{1-\sigma}-1}{1-\sigma}-P^{2} / 2\right) d t
$$

subject to (3), (5), (6). The difference to the competitive economy is that now both the pollution externality and the externality of consuming the dirty good, i.e. the habit 
formation, are taken into account in setting up the intertemporal optimization problem. To solve the optimization problem, we set up the current-value Hamiltonian. The latter is written as

$$
\begin{aligned}
\mathcal{H}^{s}= & U(\cdot)+\lambda_{1}^{s}\left(K^{\beta}-p_{d} C_{d}-p_{c} C_{c}-\delta K\right)+ \\
& \lambda_{2}^{s}\left(\omega_{1} C_{d}+\omega_{2} C_{c}-\xi P\right)+\lambda_{3}^{s} \chi \gamma\left(C_{d}-H\right),
\end{aligned}
$$

with the utility function $U(\cdot)$ given by $(1)$ and $\lambda_{i}^{s}, i=1,2,3$, the shadow prices or co-state variables of capital, pollution and of habits, respectively.

The necessary optimality conditions for an optimum are as follows

$$
\begin{aligned}
\frac{\partial \mathcal{H}}{\partial C_{d}} & =0 \leftrightarrow C_{d}=\left(\frac{(1+H)^{\chi}}{\lambda_{1} p_{d}-\lambda_{3} \gamma-\lambda_{2} \omega_{1}}\right)^{1 / \sigma}(1+H)^{-\chi} \\
\frac{\partial \mathcal{H}}{\partial C_{c}} & =0 \leftrightarrow C_{c}=\left(\lambda_{1} p_{c}-\lambda_{2} \omega_{2}\right)^{-1 / \sigma} \\
\dot{\lambda}_{1}^{s} & =(\rho+\delta) \lambda_{1}^{s}-\lambda_{1}^{s} \beta A K^{\beta-1} \\
\dot{\lambda}_{2}^{s} & =(\rho+\xi) \lambda_{2}^{s}+P \\
\dot{\lambda}_{3}^{s} & =\chi\left((\rho+\gamma) \lambda_{3}^{s}-C_{d}^{1-\sigma}(1+H)^{-\sigma}\right)
\end{aligned}
$$

In addition, we require that the usual transversality condition must hold that can be written as, $\lim _{t \rightarrow \infty} e^{-\rho t}\left(\lambda_{1}^{s} K+\lambda_{2}^{s} P+\lambda_{3}^{s} H\right)=0$. Thus, in the case of habit formation, i.e. for $\chi=1$, the economy is completely described by the differential equations (3), (5), (6), (21), (22), (23), with $C_{c}$ and $C_{d}$ determined by (19)-(20) respectively, and where $\lambda_{i}^{s}(0), i=1,2,3$, are free. ${ }^{5}$ When habit formation does not occur, i.e. for $\chi=0$, the economy is described by the differential equations (5), (6), (21), (22) and the corresponding transversality condition.

Since the competitive economy and the social optimum do not coincide, we first derive the tax rates on the polluting and on the non-polluting consumption good such that

\footnotetext{
${ }^{5}$ For $1 / \sigma \leq 2$, the utility function and, thus, the Hamiltonian is jointly concave in the control and state variables so that the necessary optimality conditions are also sufficient.
} 
the competitive economy replicates the social optimum. To do so, we denote by $\tau_{d}$ the Pigou tax rate on the dirty consumption good and by $\tau_{c}$ the Pigou tax rate on the clean good. ${ }^{6}$ The budget constraint of the household in the competitive economy, then, becomes $\dot{K}=A K^{\beta}-\left(p_{d}+\tau_{d}\right) C_{d}-\left(p_{c}+\tau_{c}\right) C_{c}-\delta K+\Gamma$, with $\Gamma$ lump-sum transfers or a lump-sum tax that are adjusted such that the budget of the government is balanecd at each point in time. Proposition 5 gives the Pigou tax rates.

Proposition 5 The competitive economy replicates the social optimum if the tax rates are set such that $\tau_{c}=\left(-\lambda_{2}^{s}\right) \omega_{2} / \lambda_{1}$ and $\tau_{d}=\left(-\lambda_{2}^{s}\right) \omega_{1} / \lambda_{1}^{s}-\chi \lambda_{3}^{s} \gamma / \lambda_{1}^{s}$ holds, with $\lambda_{1}(0)=\lambda_{1}^{s}(0)$.

Proof: See appendix D.

Proposition 5 demonstrates that the Pigou tax rate on the clean consumption good is positive since the clean consumption good also contributes to environmental pollution. It depends on the absolute value of the shadow price of pollution multiplied by the contribution of one unit of clean consumption to pollution growth, relative to the shadow price of physical capital. Only in case the clean consumption good does not lead to environmental pollution, i.e. when $\omega_{2}=0$, the optimal tax rate equals zero. In that case clean consumption does not go along with externalities so that there is no need to impose a tax on that good.

The Pigou tax rate on the dirty consumption good also depends on the absolute value of the shadow price of pollution multiplied by the contribution of one unit of dirty consumption to pollution growth, relative to the shadow price of physical capital. However, in contrast to the optimal tax rate on the clean consumption good, the Pigou tax on the dirty consumption good is also a function of habits. The stronger the habit formation and its shadow price are, the lower is the optimal tax rate on the dirty good. If habit formation is very strong, the tax rate on the dirty consumption good may even be negative meaning that the government pays a subsidy for the polluting good. In that case, the consumption

\footnotetext{
${ }^{6}$ We consider a quantity tax but an ad-valorem tax would would lead to the same result.
} 
of the dirty good in the competitive economy is too low compared to the social optimum. This also implies that pollution at the steady-state in the competitive economy without government intervention is lower than in the social optimum. The reason for that outcome is to be seen in the second externality in this model, namely in habit formation that has a positive effect on utility. Since that effect is not taken into account in the competitive economy, the consumption of the dirty good in the social optimum can be higher than in the competitive economy. This will occur when habit formation and its shadow value are large.

If, on the other hand, the consumption of the dirty good in the competitive economy is too high, the Pigou tax rate on the polluting good will be strictly positive. This is the case when habit formation is not very strong so that the social planner does not attach a high importance on that phenomenon but puts more emphasis on the negative externality going along with consumption, i.e. on pollution control. More conceretly, this will always hold when the cost of consuming one unit of the dirty good exceeds its external benefits that consist in raising the stock of habits. In the case of no habit formation, we get the usual result that the steady-state pollution in the competitive economy is higher than in the social optimum. We summarize these results in the following proposition 6 .

Proposition 6 Without habit formation, the steady-state pollution in the competitive economy exceeds the steady-state pollution in the social optimum. With habit formation, the steady-state pollution in the competitive economy falls short of the steady-state pollution in the social optimum if and only if $\left(\lambda_{3}^{s}\right)^{\star} \gamma>\left(-\lambda_{2}^{s}\right)^{\star} \omega_{1}$ holds.

Proof: See appendix E.

As concerns existence, uniqueness and stability of the steady-state, it is difficult to make statements for the analytical model due to its complexity. However, for the model without habit formation it is possible to show that the steady-state of the social optimum is a saddle point. This is the contents of proposition 7 . 
Proposition 7 In the social optimum without habit formation, the steady-state is saddle point stable.

Proof: See appendix F.

To gain additional insight and in order to illustrate our analytical results, we resort to the numerical example presented above. Table 2 presents the steady-state values for the social optimum without and with habits. ${ }^{7}$ As in the competitive economy, the presence of habits raises the ratio of clean to dirty consumption and, thus, reduces steady-state pollution if the preferences are characetrized by distant complementarity. In the case of adjacent complementarity, the revers holds. Then, habits reduce clean consumption relative to the dirty one and lead to higher pollution.

\begin{tabular}{|c|c|c|c|c|c|}
\hline & \multicolumn{2}{|c|}{ No habits $(\chi=0)$} & \multicolumn{3}{|c|}{ With habits $(\chi=1)$} \\
\hline & $C_{c}^{\star} / C_{p}^{\star}$ & $P^{\star}$ & $C_{c}^{\star} / C_{p}^{\star}$ & $P^{\star}$ & $H^{\star}$ \\
\hline $1 / \sigma=1 / 2$ & 1.084 & 0.650 & 1.207 & 0.621 & 0.554 \\
\hline $1 / \sigma=4 / 3$ & 1.419 & 0.577 & 0.859 & 0.714 & 0.658 \\
\hline
\end{tabular}

Table 2: Steady-state values of the social optimum $\left(K^{\star}=4.192\right)$.

Comparing the competitive economy with the social optimum when habit formation is present, one realizes that in the case of distant complementarity the ratio of clean consumption relative to the dirty one in the social optimum is smaller than in the competitive economy leading to a higher steady-state pollution and to a higher stock of habits. The reason for that outcome lies in the benefits of habit formation that exceed the costs of pollution. The optimal steady-state tax rates for the economy in this case are $\tau_{c}=0.021$ and $\tau_{d}=-0.043$ showing that the government in the competitive should subsidize the

\footnotetext{
${ }^{7}$ Again, there exists a unique saddle point stable steady-state with two and three negative real eigenvalues, respectively.
} 
dirty consumtpion good. In the case of adjacent complementarity, the steady-state pollution and the stock of habits in the social optimum are lower compared to the competitive economy and the ratio of clean to dirty consumption is higher. The Pigou tax rates at the steady-state for this case are $\tau_{c}=0.036$ and $\tau_{d}=0.049$ showing that both goods should be taxed in the competitive economy.

\section{Conclusion}

In this paper we have studied the effects of habit formation on pollution for an otherwise standard exogenous growth model, where the consumer can choose between a conventional dirty good and a good that contributes less to pollution. Habits arise from the past consumption of the polluting good and are defined as routines that result from unintended learning processes and that simplify everday life.

The analysis of the competitive model economy has shown that the presence of habits raises the consumption of the clean good relative to the dirty good if the preferences are characterized by distant complementarity and reduces that ratio if there is adajacent complementarity. Further, the effects of habits on the steady-state pollution stock depend on whether distant or adjacent complementarity is given and on the contribution of the goods to pollution as well as on their relative price. The Pigou tax rates have been derived and it turned out that pollution in the social optimum can be higher than in the competitive economy if habit formation is sufficiently strong so that the Pigou tax on the dirty good is negative, i.e. the optimal policy is to subsidize the dirty consumption good.

This paper is a first approach to study the role of preferences with regard to consumer behaviour and with regard to pollution. Hence, it could be extended in several directions. For example, one could resort to a more general CES utility function where clean and dirty consumption goods are not perfect substitutes. Further, the prices of the two types of goods have been assumed to be constant parameters. Thus, it would be interesting to 
allow for learning effects in the production of the less polluting good, for example, that reduce its price over time. Finally, it would also be interesting to analyze that model for the case of ongoing growth that is generated endogenously.

\section{A Proof of proposition 2}

At the steady-state, we have $p_{c} C_{c}^{\star}+p_{d} C_{d}^{\star}=A\left(K^{\star}\right)^{\beta}-\delta K^{\star}=$ constant, with $K^{\star}=$ $(A \beta /(\rho+\delta))^{1 /(1-\beta)}$ obtained from $\dot{\lambda}_{1}=0$. This gives $C_{d}^{\star}=\left(A\left(K^{\star}\right)^{\beta}-\delta K^{\star}-p_{c} C_{c}^{\star}\right) / p_{d}$. Thus, the pollution in steady-state is obtained from $\dot{P}=0$ as

$$
P^{\star}=\frac{\omega_{1}\left(A\left(K^{\star}\right)^{\beta}-\delta K^{\star}\right)}{\xi p_{d}}+\frac{\omega_{1} p_{c} C_{c}^{\star}}{\xi p_{d}}-\frac{\omega_{2} C_{c}^{\star}}{\xi}
$$

giving

$$
\frac{d P^{\star}}{d C_{c}^{\star}}=\frac{1}{\xi}\left(\omega_{2}-\frac{p_{c} \omega_{1}}{p_{d}}\right)>(<) 0 \leftrightarrow \omega_{2} p_{d}>(<) \omega_{1} p_{c}
$$

From $\dot{K}=0$, one obtains for the model with habits $(\chi=1)$

$$
\lambda_{1}^{\star}=\left(\frac{(1+H)^{(1-\sigma) / \sigma} p_{d}^{1-1 / \sigma}+p_{c}^{1-1 / \sigma}}{A\left(K^{\star}\right)^{\beta}-\delta K^{\star}}\right)^{-\sigma}
$$

and

$$
\frac{d \lambda_{1}^{\star}}{d H}=\frac{(1-\sigma)\left(\lambda_{1}^{\star}\right)^{\sigma-1}(1+H)^{(1 / \sigma)-2} p_{d}^{1-1 / \sigma}}{A\left(K^{\star}\right)^{\beta}-\delta K^{\star}}>(<) 0 \leftrightarrow 1 / \sigma>(<) 1
$$

Since $d C_{c} / d \lambda_{1}<0$, this shows that $d C_{c} / d H>(<) 0$ for $1 / \sigma<(>) 1$. Noting that for $1 / \sigma<(>) 1$ the preferences are characterized by distant (adjacent) complementarity, this proves the proposition.

\section{B Proof of proposition 3}

Setting $\dot{\lambda}_{1}=0$ gives $K^{\star}=(A \beta /(\rho+\delta))^{1 /(1-\beta)}$. From $\dot{K}=0$, one obtains for the model without habits $(\chi=0)$

$$
\lambda_{1}^{\star}=\left(\frac{A\left(K^{\star}\right)^{\beta}-\delta K^{\star}}{p_{d}^{1-1 / \sigma}+p_{c}^{1-1 / \sigma}}\right)^{-\sigma}
$$


Note that $A\left(K^{\star}\right)^{\beta}>\delta K^{\star}$ holds because $A\left(K^{\star}\right)^{\beta} \leq \delta K^{\star}$ would imply $\delta \geq(\rho+\delta) / \beta$, which follows from $K^{\star}=(A \beta /(\rho+\delta))^{1 /(1-\beta)}$. That, however, is excluded because of $\beta \in(0,1)$ and $\rho>0$.

Finally, $\dot{P}=0$ leads to

$$
P^{\star}=\xi^{-1}\left(\frac{\omega_{1}}{\left(\lambda_{1}^{\star} p_{d}\right)^{1 / \sigma}}+\frac{\omega_{2}}{\left(\lambda_{1}^{\star} p_{c}\right)^{1 / \sigma}}\right)
$$

For $\chi=0$, the Jacobian matrix of (12)-(14) is given by

$$
\mathrm{J}=\left[\begin{array}{ccc}
\rho+\delta-A \beta K^{\beta-1} & -\lambda_{1} A \beta(\beta-1) K^{\beta-2} & 0 \\
\sigma^{-1} \lambda_{1}^{-1-1 / \sigma}\left(p_{d}^{1-1 / \sigma}+p_{c}^{1-1 / \sigma}\right) & A \beta K^{\beta-1}-\delta & 0 \\
-\sigma^{-1} \lambda_{1}^{-1-1 / \sigma}\left(\frac{\omega_{1}}{\left(\lambda_{1}^{\star} p_{d}\right)^{1 / \sigma}}+\frac{\omega_{1}}{\left(\lambda_{1}^{\star} p_{c}\right)^{1 / \sigma}}\right) & 0 & -\xi
\end{array}\right]
$$

with $K$ and $\lambda_{1}$ evaluated at the rest point $\left\{K^{\star}, \lambda_{1}^{\star}\right\}$.

One eigenvalue of that matrix is $\mu_{1}=-\xi$. The other two are given by the eigenvalues of

$$
\mathrm{J}^{p}=\left[\begin{array}{cc}
\rho+\delta-A \beta K^{\beta-1} & -\lambda_{1} A \beta(\beta-1) K^{\beta-2} \\
\sigma^{-1} \lambda_{1}^{-1-1 / \sigma}\left(p_{d}^{1-1 / \sigma}+p_{c}^{1-1 / \sigma}\right) & A \beta K^{\beta-1}-\delta
\end{array}\right]
$$

Using that $\rho+\delta-A \beta K^{\beta-1}=0$ holds at the steady-state, the determinant of $\mathrm{J}^{p}$ can be computed as

$$
\operatorname{det} \mathrm{J}^{p}=\lambda_{1} A \beta(\beta-1) K^{\beta-2} \sigma^{-1} \lambda_{1}^{-1-1 / \sigma}\left(p_{d}^{1-1 / \sigma}+p_{c}^{1-1 / \sigma}\right)<0
$$

Hence, one eigenvalue of $\mathrm{J}^{p}$ is negative and one is positive so that $J$ has two negative eigenvalues and one positive yielding saddle point stability.

\section{Proof of proposition 4}

Setting $\dot{\lambda}_{1}=0$ again yields $K^{\star}=(A \beta /(\rho+\delta))^{1 /(1-\beta)}$. Solving $\dot{H}=0$ gives $\lambda_{1}^{\star}=$ $p_{d}^{-1}\left(\gamma H(1+H)^{1-1 / \sigma}\right)^{-\sigma}$. Inserting $K^{\star}$ and $\lambda_{1}^{\star}$ in $\dot{K}$ leads to

$$
\dot{K}=A\left(K^{\star}\right)^{\beta}-\delta K^{\star}-H-p_{c}\left(\frac{p_{d}}{p_{c}}\right)^{1 / \sigma} H(1+H)^{1-1 / \sigma}
$$


The derivative of $\dot{K}$ is

$$
\frac{\partial \dot{K}}{\partial H}=-1-p_{c}\left(\frac{p_{d}}{p_{c}}\right)^{1 / \sigma}(1+H)^{-1 / \sigma}(1+2 H-H / \sigma)
$$

Thus, the function $\dot{K}$ starts at a positive value for $H=0$, is monotonically declining in $H$, for $1 / \sigma \leq 2$, and converges to $-\infty$ for $H \rightarrow \infty$. Consequently, there exists a unique positive finite $H^{\star}$ that solves $\dot{K}=0$. Finally, solving $\dot{P}=0$ one obtains

$$
P^{\star}=\left(\omega_{1} / \xi\right)\left(\frac{\left(1+H^{\star}\right)^{1-\sigma}}{\lambda_{1}^{\star} p_{d}}\right)^{1 / \sigma}+\left(\omega_{2} / \xi\right)\left(\lambda_{1}^{\star} p_{c}\right)^{-1 / \sigma}
$$

For $\chi=1$, the Jacobian matrix of (12)-(15) is given by

$$
\mathrm{J}_{1}=\left[\begin{array}{cccc}
-A \beta K^{\beta-1}+\delta+\rho & -A(\beta-1) \beta K^{\beta-2} \lambda_{1} & 0 & 0 \\
\frac{p_{c}{ }^{2}\left(\lambda_{1} p_{c}\right)^{-1-\frac{1}{\sigma}}}{\sigma}+\frac{(H+1)^{\frac{1}{\sigma}-1} p_{d}{ }^{2}\left(\lambda_{1} p_{d}\right)^{-1-\frac{1}{\sigma}}}{\sigma} & A \beta K^{\beta-1}-\delta & 0 & -C_{1} \\
-\frac{p_{c} \omega_{2}\left(\lambda_{1} p_{c}\right)^{-1-\frac{1}{\sigma}}}{\sigma}-\frac{(H+1)^{\frac{1}{\sigma}-1} p_{d}\left(\lambda_{1} p_{d}\right)^{-1-\frac{1}{\sigma} \omega_{1}}}{\sigma} & 0 & -\xi & \omega_{1} C_{1} \\
-\frac{\gamma(H+1)^{\frac{1}{\sigma}-1} p_{d}\left(\lambda_{1} p_{d}\right)^{-1-\frac{1}{\sigma}}}{\sigma} & 0 & 0 & \gamma\left(C_{1}-1\right)
\end{array}\right],
$$

with $C_{1}=(H+1)^{(1 / \sigma)-2} p_{d}\left(\lambda_{1} p_{d}\right)^{-1 / \sigma}((1 / \sigma)-1)$ and $K, \lambda_{1}, P$ and $H$ evaluated at the rest point $\left\{K^{\star}, \lambda_{1}^{\star}, P^{\star}, H^{\star}\right\}$.

One eigenvalue of that matrix is $\mu_{1}=-\xi$. The other three eigenvalues are the eigenvalues of

$$
\mathrm{J}_{1}^{p}=\left[\begin{array}{ccc}
A \beta K^{\beta-1}-\delta & -C_{1} & \frac{p_{c}{ }^{2}\left(\lambda_{1} p_{c}\right.}{\sigma}+\frac{(H+1)^{\frac{1}{\sigma}-1} p_{d}{ }^{2}\left(\lambda_{1} p_{d}\right)^{-1-\frac{1}{\sigma}}}{\sigma} \\
0 & \gamma\left(C_{1}-1\right) & -\frac{\gamma(H+1)^{\frac{1}{\sigma}-1} p_{d}\left(\lambda_{1} p_{d}\right)^{-1-\frac{1}{\sigma}}}{\sigma} \\
-A(\beta-1) \beta K^{\beta-2} \lambda_{1} & 0 & -A \beta K^{\beta-1}+\delta+\rho
\end{array}\right]
$$

Using that $\rho+\delta-A \beta K^{\beta-1}=0$ holds at the steady-state, the determinant of $\mathrm{J}_{1}^{p}$ can be computed as

$$
\begin{aligned}
& \operatorname{det} J_{1}^{p}= \frac{A(1-\beta) \beta K^{\beta-2}\left(\lambda_{1} p_{c}\right)^{-1 / \sigma}\left(\lambda_{1} p_{d}\right)^{-1 / \sigma}\left(\gamma p_{c}(\sigma-1)(H+1)^{\frac{1}{\sigma}}\right)}{(H+1)^{2} \sigma^{2}}+ \\
& \frac{A(1-\beta) \beta K^{\beta-2}\left(\gamma\left(p_{d}(H+1)^{\frac{1}{\sigma}}\left(\lambda_{1} p_{c}\right)^{\frac{1}{\sigma}}+(H+1) p_{c}\left(\lambda_{1} p_{d}\right)^{\frac{1}{\sigma}}\right)\right)}{\left(\lambda_{1} p_{c}\right)^{1 / \sigma}\left(\lambda_{1} p_{d}\right)^{1 / \sigma}(H+1) \sigma}
\end{aligned}
$$


so that $1 / \sigma \leq 1$ is sufficient but not necessary for $\operatorname{det} J_{1}^{p}>0$. Next, we define

$$
W_{1}=a_{11} a_{22}-a_{12} a_{21}+a_{22} a_{33}-a_{23} a_{32}+a_{11} a_{33}-a_{13} a_{31},
$$

with $a_{i j}$ the element of the $i$-th row and $j$-th column of the matrix $\mathrm{J}_{1}^{p}$. In our case, we get

$$
W_{1}=\left(A \beta K^{\beta-1}-\delta\right) \gamma\left(C_{1}-1\right)-A \beta(1-\beta) K^{\beta-2} \lambda_{1}^{c} a_{13}
$$

From $\rho+\delta-A \beta K^{\beta-1}=0$ we know that $A \beta K^{\beta-1}-\delta>0$ holds. Recalling that $C_{1}=$ $(H+1)^{(1 / \sigma)-2} p_{d}\left(\lambda_{1} p_{d}\right)^{-1 / \sigma}((1 / \sigma)-1)$ and using $a_{13}>0$ shows that $1 / \sigma \leq 1$ is a sufficient but not necessary condition for $W_{1}<0$. Since $\operatorname{det} J_{1}^{p}>0$ and $W_{1}<0$ are sufficient for two negative and and one positive eigenvalue of $J_{1}^{p}$ (see Wirl, 1997), this demonstrates that $1 / \sigma \leq 1$ is a sufficient but not necessary condition for $\mathrm{J}_{1}$ to have one positive and three negative eigenvalues.

\section{Proof of proposition 5}

We equate (9) with (19) to obtain the optimal tax rate on the dirty good and (10) with (20) to obtain the optimal tax rate on the clean consumption good, with $p_{d}+\tau_{d}$ the price of the dirty good in the competitive economy and $p_{c}+\tau_{c}$ the price of the clean good in the competitive economy. Further, setting the initial shadow prices of physical capital to the same value, i.e. $\lambda_{1}(0)=\lambda_{1}^{s}(0)$, assures that the shadow price of capital in the competitive economy is equal to that in the social optimum for all $t \in[0, \infty)$.

\section{E Proof of proposition 6}

To prove that proposition, we note that in the economy without habit formation the relative steady-state consumption is given by $C_{r e l}=p_{c} / p_{d}$ for the competitive economy. In the social optimum it is given by

$$
C_{r e l}^{s}=\frac{p_{c}-\omega_{2} \lambda_{2}^{s} / \lambda_{1}^{s}}{p_{d}-\omega_{1} \lambda_{2}^{s} / \lambda_{1}^{s}}
$$


Thus, steady-state consumption of the clean good relative to the dirty good in the competitive economy is always lower than in the social optimum because of $\omega_{2}<\omega_{1}$ and $\lambda_{2}<0$. Since total consumption is fix at the steady-state, this implies that dirty consumption is higher and clean consumption is lower so that the steady-state stock of pollution in the competitive economy is larger.

With habit formation, the tax rate on the dirty good is negative if the condition in the proposition holds. A negative tax implies that consumption of the dirty good in the competitive economy without government intervention is lower than in the social optimum. Together with a fixed total steady-state consumption this implies that the steady-state pollution in the competitive economy is lower than in the social optimum.

\section{F $\quad$ Proof of proposition 7}

To prove that proposition we note that the dynamics of the social optimum without habit formation is described by the equations (5), (6), (21) and (22). The Jacobian for that dynamic system is

$$
\mathrm{J}_{2}=\left(\begin{array}{cccc}
A \beta K^{\beta-1}-\delta & 0 & \frac{p_{c}{ }^{2} C_{c}^{-1}}{\sigma}+\frac{p_{d}{ }^{2} C_{d}^{-1}}{\sigma} & -\frac{p_{c} \omega_{2} C_{c}^{-1}}{\sigma}-\frac{p_{d} \omega_{1} C_{d}^{-1}}{\sigma} \\
0 & -\xi & -\frac{p_{c} \omega_{2} C_{c}^{-1}}{\sigma}-\frac{p_{d} \omega_{1} C_{d}^{-1}}{\sigma} & \frac{\omega_{2}{ }^{2} C_{c}^{-1}}{\sigma}+\frac{\omega_{1}{ }^{2} C_{d}^{-1}}{\sigma} \\
-A(\beta-1) \beta \lambda_{1} K^{\beta-2} & 0 & -A \beta K^{\beta-1}+\delta+\rho & 0 \\
0 & 1 & 0 & \rho+\xi
\end{array}\right),
$$

with $C_{c}=\left(\lambda_{1} p_{c}-\lambda_{2} \omega_{2}\right)^{-1 / \sigma}$ and $C_{d}=\left(\lambda_{1} p_{d}-\lambda_{2} \omega_{1}\right)^{-1 / \sigma}$. Using $\rho+\delta=A \beta K^{\beta-1}$ at the steady-state that follows from $\dot{\lambda}_{1}=0$, the determinant can be computed as $\operatorname{det} \mathrm{J}_{2}=A(1-\beta) \beta \lambda_{1} K^{\beta-2}\left(\frac{\xi(\rho+\xi)\left(p_{c}{ }^{2} C_{c}^{-1}+p_{d}{ }^{2} C_{d}^{-1}\right)}{\sigma}+\frac{\left(p_{c} \omega_{1}-p_{d} \omega_{2}\right)^{2} C_{c}^{-1} C_{d}^{-1}}{\sigma^{2}}\right)>0$ Next, we define $W_{2}$ as

$$
W_{2}=a_{11} a_{33}-a_{13} a_{31}+a_{22} a_{44}-a_{24} a_{42}+2\left(a_{12} a_{34}-a_{14} a_{32}\right)
$$


For our model, $W_{2}$ is computed as

$$
W_{2}=A(\beta-1) \beta \lambda_{1} K^{\beta-2}\left(\frac{p_{c}^{2}}{C_{c} \sigma}+\frac{p_{d}^{2}}{C_{d} \sigma}\right)-\xi(\rho+\xi)-\frac{\omega_{2}^{2}}{C_{c} \sigma}-\frac{\omega_{1}^{2}}{C_{d} \sigma}<0
$$

According to lemma 2 in Dockner and Feichtinger (1991), a positive determinant and a negative $W_{2}$ implies two negative real eigenvalues or two complex conjugate eigenvalues with negative real parts. This proves the proposition.

\section{References}

Aronson, E., Wilson, T.D. and R.M. Akert (2004) Social Psychology. 4th ed., Pearson Education, Prentice Hall.

Dockner, E.J. and G. Feichtinger (1991) "On the optimality of limit cycles in dynamic economic systems." Journal of Economics, Vol. 53: 31-50.

Greiner, A., (1998) Fiscal Policy and Economic Growth. Ashgate Publishing Company, Aldershot (2nd printing).

Greiner, A., and W. Semmler (2008) The Global Environment, Natural Resources and Economic Growth. Oxford University Press, Oxford, New York.

Greiner, A., L. Grüne and W. Semmler (2014) "Economic growth and the transition from non-renewable to renewable energy. "Environment and Development Economics, Vol. 19: 417-439.

Havranek, T., R. Horvath, Z. Irsova and M. Rusnak (2015) "Cross-country heterogeneity in intertemporal substitution", Journal of International Economics, Vol. 96: 100-118.

Hodgson, G.M. (2004) "Reclaiming habit for institutional economics. Journal of Economic Psychology, Vol. 25: 651-660. 
Hoel, M., and S. Kverndokk (1996) "Depletion of fossil fuels and the impacts of global warming." Resource and Energy Economics, Vol. 18:115-136.

Ikefuji, M. (2008) "Habit formation in an endogenous growth model with pollution abatement activities." Journal of Economics, Vol. 94: 241259.

Krautkraemer, J.A. (1998) "Nonrenewable resource scarcity." Journal of Economic Literature, Vol. 36: 2065-107.

Orchecchia, C. and M.L. Tessitore (2011) "Economic growth and the environment with clean and dirty consumption." Fondazione Eni Enrico Matteo, Nota di Lavora 57.2011, Milano.

Ryder, H.E. and G.M. Heal (1973) "Optimal growth with intertemporally dependent preferences." Review of Economic Studies, Vol. 40: 1-31.

Scalera, D. (1996) "Optimal consumption and the environment: Choosing between 'clean' and 'dirty' goods." Environmental and Resource Economics, Vol. 7: 375-389.

van der Ploeg, F. and C. Withagen (2014) "Growth, renewables and the optimal carbon tax." International Economic Review, Vol. 55: 283-311.

Wirl, F. (1997) "Stability and limit cycles in one-dimensional dynamic optimisations of competitive agents with a market externality." Journal of Evolutionary Economics, Vol. 7: 73-89. 